\title{
Aplicando o group storytelling no compartilhamento de experiências e na avaliação em sala de aula
}

\author{
Bruno B. Lage ${ }^{1,2}$, Juliana B. S. França ${ }^{1}$, Angélica F. S. Dias ${ }^{1}$, \\ Adriana S. Vivacqua ${ }^{1}$, Marcos R. S. Borges ${ }^{1}$
}

${ }^{1}$ Programa de Pós-Graduação em Informática - Universidade Federal do Rio de Janeiro (UFRJ)

Caixa Postal 68.530 - 21.941-590 - Rio de Janeiro, RJ - Brazil

${ }^{2}$ Departamento de Ciência da Computação

Campus Engenho Novo II - Colégio Pedro II (CPII) - Rio de Janeiro, RJ - Brazil

brunobarboza@gmail.com, \{juliana.franca, angelica,mborges\}@ppgi.ufrj.br, avivacqua@dcc.ufrj.br

\begin{abstract}
Telling a story is a great opportunity to teach one who seeks knowledge or aims at building it. However, when told in group, the story has the potential of various visions and opinions, it is much richer in knowledge and insights. In the classroom, it is a great way to transfer knowledge, but when it is sought to verify the learning and acceptance of teaching methods and learning objects, a story collaboratively constructed by students can prove to be an efficient tool for teachers. This research is dedicated to applying the technique of group storytelling in the validation of the contents learned in a project-oriented discipline of an undergraduate course. The stories collaboratively built by students were analyzed and discussed by the teachers for extracting information that validates or refutes the structuring and content of the analyzed course. The findings show that the technique is feasible to the discipline as an instrument for evaluation and improvement, but needs to be ongoing and not a one-off exercise.
\end{abstract}

Resumo. Contar uma história é uma ótima oportunidade de ensinar àquele que procura o conhecimento ou busca construí-lo. Porém, quando contada em grupo a história tem o potencial de várias visões e opiniões, com conhecimentos e percepções muito maiores incutidos. Em sala de aula, ela é um ótimo meio de transferir conhecimento, mas quando busca-se verificar o aprendizado, bem como a aceitação de métodos de ensino e de objetos de aprendizagem, a história construída de forma colaborativa pelos alunos pode mostrar-se um instrumento eficiente para os professores. Esta pesquisa aplica a técnica de group storytelling na validação da aprendizagem dos conteúdos em uma disciplina orientada à projetos da graduação. As histórias construidas colaborativamente pelos alunos da disciplina foram analisadas e discutidas pelos docentes, com o objetivo de extrair informações que validem ou refutem a estruturação e conteúdo da disciplina. As conclusões mostram que a técnica é viável para a disciplina como instrumento de avaliação e aprimoramento, mas precisa ser um exercício contínuo e não pontual.

\section{Introdução}

Histórias estruturam a sociedade e são usadas para definir cada um como indivíduo ou com um grupo, conectando ao passado, direcionando para o futuro e onde cada 
elemento representa a interseção de várias outras histórias, mesclando fato e ficção [Sax 2006]. Elas representam o mecanismo natural mais antigo do desenvolvimento humano que permitiu às pessoas passarem informações, conceitos, procedimentos e comportamentos de grupos pequenos, como famílias, até grandes grupos, como clãs e nações [Haigh e Hardy 2011, Acosta et al. 2004].

Atualmente, vivemos o momento da era da informação e da integração impulsionada pela Internet e os dispositivos móveis, uma verdadeira revolução na vida dos cidadãos, conhecida como: Revolução da Internet, Revolução Digital, dentre outras [Pimentel e Fuks 2012]. A Geração Y, Digital ou da Internet, apesar de todo o potencial, ainda utiliza expressões de comunicação com o mundo — principalmente através das mídias sociais e blogs — que muito se assemelham aos mecanismos utilizados na estruturação de histórias. Essa comunicação gera textos e outras mídias que refletem momentos, pensamentos, ideologias, entre outros, com diferentes pontos de vista sobre um mesmo assunto. E os jovens deste século trabalham todo o tempo conectados, compartilhando informação, atuando em conjunto e aprendendo com a colaboração das demais pessoas. Com isso, eles passaram a adotar uma postura investigativa diante das informações recebidas e a forma de aprender foi inteiramente modificada.

Se essas formas de absorver conhecimento mudaram com as tecnologias; em contrapartida as tecnologias também passaram a ser incluídas no ambiente educativo. Por muitos anos, o uso de novas tecnologias em sala de aula e a introdução de outros meios, tais como vídeos, jogos, video games e outras aplicações, no processo de aprendizagem mostraram-se mecanismos viáveis para atingir níveis satisfatórios de motivação do aprendizado [Padilla-Zea et al. 2014].

Essa mudança no ambiente educacional é reflexo da adaptação dos conteúdos ao novo momento dos alunos formados a partir dessa nova geração; talvez muito mais mudanças surjam e ocorram deveras rápido. Paralelamente a isso, a forma de transferir o conteúdo vem sendo trabalhada com muita criatividade e em novos materiais e objetos de aprendizagem [Guterres e Silveira 2016, Tekkumru Kisa e Stein 2015, Molina et al. 2014, Fink 2013]. Entretanto, se a passagem dos conteúdos vem sendo fomentada por grandes novidades, a busca pela verificação e análise desses conteúdos que foram de fato apreendidos pelos alunos é um ainda desafio a ser enfrentado.

Os métodos tradicionais de avaliação vêm mudando constantemente para adequar-se à realidade de que os alunos também mudaram e continuam mudando, o que exige dos professores e instituições novos mecanismos, técnicas e/ou metodologias. Pensando nisso, e tendo certeza do potencial das histórias em transferir conhecimento, este trabalho busca a via contrária: não contar a história, mas construí-la - e de forma colaborativa. Ou seja, ao invés da história ser utilizada como mecanismo de transferência de informações somente, criada por terceiros e já verificada, ela seria construída pelos alunos e serviria de verificação da retenção dos conceitos apresentados na disciplina. Além disso, a história seria também uma forma de levantar as impressões e aceitação dos alunos quanto aos objetos de aprendizagem usados ao longo da disciplina e a abordagem de ensino proposta. Uma vez que as histórias sejam uma forma eficaz de captura da percepção e aceitação dos alunos sobre uma disciplina, esses resultados podem se tornar efetivos para os professores repensarem sobre suas atividades em docência considerando seu público alvo. 
Esta pesquisa foi realizada com alunos de graduação, do curso de Ciência da Computação, dentro de uma disciplina orientada à projetos e que defende o trabalho colaborativo entre os alunos. Nela viu-se a necessidade da obtenção de um retorno por parte desses alunos a fim de levantar o que por eles foi percebido e aceito sobre (i) os conteúdos apresentados ao longo da disciplina, (ii) a forma de condução das aulas e (iii) o método colaborativo de ensino. Para isso, foi utilizada a técnica de group storytelling, que usa histórias construídas em grupo para a formalização do conhecimento tácito e estruturadas como fragmentos (narrativas) que podem ser associadas entre si, formando um encadeamento de ideias, percepções e visões dos vários participantes. Esta estratégia é inspirada em Valle et al. (2002) e em Lage e Quintanilha (2010).

O presente artigo é organizado da seguinte forma: a Seção 2 trata brevemente de alguns trabalhos relacionados ao uso do storytelling em sala de aula. A Seção 3 apresenta a metodologia de pesquisa proposta, a condução do desenvolvimento da pesquisa, dados coletados e discussões dos resultados. A quarta e última seção discute as conclusões alcançadas nesta investigação e os próximos passos da pesquisa.

\section{Trabalhos Relacionados}

A atual pesquisa foca na utilização do storytelling em grupo em sala de aula como meio de avaliar os alunos. Mais precisamente, para esse fim, utilizou-se o group storytelling, uma técnica focada na recuperação de conhecimentos tácitos e informações aplicada mais fortemente em ambientes corporativos - maiores detalhes sobre a técnica serão discutidos na próxima sessão. Portanto, observaremos nos trabalhos a seguir a utilização das histórias e sua aplicação na avaliação dos docentes, sem necessariamente explorar o aparato tecnológico empregado.

Hoje em dia, o número de trabalhos e metodologias voltados para docentes criarem histórias como ferramenta de ensino (história criada pelo professor) é muito maior do que os que exploram a criação de histórias pelos alunos, muito menos os que utilizam as histórias criadas pelos discentes como meio de análise do desenvolvimento deles. Focado nestes últimos, pode-se destacar o de Krom e Williams (2011) cujo objetivo foi criar histórias, como em um conto de fadas, que explorassem os princípios de contabilidade ensinados a alunos do primário que estão aprendendo esse conteúdo. $\mathrm{O}$ princípio básico era explorar a criatividade e a capacidade de associação do aluno que, tentando explicar as bases da contabilidade em um formato lúdico, acabava por apreciar melhor o conteúdo. As histórias criadas eram lidas pelos docentes que interpretavam e avaliavam por critérios livres.

Já o trabalho de Santos Green (2013) reflete a estratégia típica do ensino de línguas que é a escrita de algum texto ou redação utilizadas para mensurar o aprendizado. Neste caso específico, ele usa o digital storytelling — uma forma de contação de histórias em que as pessoas usam algum tipo de ferramental digital e contam sua história, tal como acontece em mídias sociais e blogs - para que os alunos criem uma história. Nesta abordagem, os discentes trabalham em conjunto e individualmente, sendo de livre escolha deles, e o conteúdo é avaliado pelos docentes que utilizam os critérios de estética textual, ortografia, semântica e a combinação com outros tipos de mídias.

No trabalho de Salim (2016), os autores descrevem um experimento realizado com alunos do ensino fundamental aplicado ao aprendizado da língua inglesa. $\mathrm{O}$ 
objetivo desse trabalho foi capturar através de relatos construídos pelos alunos, a influência da abordagem comunicativa na sala de aula. Embora o artigo tenha apresentado resultados positivos na aquisição de conhecimento da língua inglesa, com o uso de uma ferramenta apoiada no storytelling, os resultados alcançados através do uso da técnica ainda necessitam de melhorias e uma aplicação mais ampla do estudo.

O trabalho que mais aproximou-se deste em termos conceituais foi o de Rambe e Mlambo (2014). Eles utilizaram abordagens com os alunos, não para construir uma história coletivamente, mas para construir um mapeamento através das histórias individuais das dificuldades encontradas por alunos com diferentes níveis educacionais que ingressavam no ensino superior e tentar usar esse conhecimento adquirido para melhorar a estrutura educacional e evitar evasões. Apesar de não construir colaborativamente a história, a análise das histórias individuais é uma forma de estimar os aspectos coletivos, ou seja, mesmo que não aplicado em sala de aula como instrumento de avaliação dos alunos, as histórias e o conhecimento fragmentado dentro delas, serviriam como métricas para o melhoramento não de uma disciplina, mas da instituição em si. São propósitos muito semelhantes ao buscados neste trabalho.

Pode-se, portanto, destacar que a principal diferença trazida por esta pesquisa em comparação com as anteriormente citadas está na construção colaborativa de histórias, por parte dos alunos, como meio de identificação das dificuldades e fatores de sucesso na condução de conteúdo em uma disciplina orientada a projetos, com o foco na aprendizagem de colaboração e apoiada por ferramental computacional.

\section{Metodologia e Desenvolvimento}

O experimento conduzido com o grupo de alunos utilizou-se das etapas descritas pela técnica de group storytelling apoiada pelo uso da ferramenta computacional Sofia 2.0 [Lage e Quintanilha 2010], e seguiu o formato exposto no Quadro 1. Ela divide a história em fragmentos associados entre si, onde um Fragmento pode ser entendido como um pedaço literal da história com a visão de alguém e que depois, junto com mais fragmentos de outras pessoas possam ser associados ou não entre si para formar a história. Isso permite à história não ter um viés somente, muito menos a visão de uma só pessoa.

Quadro 1. Estrutura da técnica de group storytelling.

\begin{tabular}{|c|c|c|}
\hline \multirow{3}{*}{ Etapas } & Pré-dinâmica & - Convocação dos participantes \\
\hline & Dinâmica & - Construção colaborativa \\
\hline & Pós-dinâmica & - Construção de uma versão escrita (alunos) \\
\hline Papéis & Dinâmicas & $\begin{array}{l}\text { - Facilitador - coordenação da construção e da finalização da história } \\
\text { - Contador - construção da história e cooperação com os demais contadores } \\
\text { - Leitor - quem extrairá do conhecimento buscado e pretendido da história }\end{array}$ \\
\hline \multicolumn{2}{|c|}{ Estruturação } & $\begin{array}{l}\text { - Fragmentos com associações e comentários e classificação por tipo } \\
\text { - Glossário } \\
\text { - Mídias (vídeos, áudios, imagens, documentos, etc.) classificadas por tipo }\end{array}$ \\
\hline \multicolumn{2}{|c|}{ Insumos e Produtos } & $\begin{array}{l}\text { - Textos (Fragmentos, comentários, glossário) } \\
\text { - Associações }\end{array}$ \\
\hline
\end{tabular}

A interação para a construção da história é o ponto chave, pois permite que os demais participantes comentem sobre o fragmento descrito e apoiem a construção de um entendimento sobre um assunto, fato ou acontecimento. Esse fator é extremamente relevante, pois segundo Souza e Schneider (2016), a aprendizagem colaborativa precisa 
VI Congresso Brasileiro de Informática na Educação (CBIE 2017)

Anais do XXIII Workshop de Informática na Escola (WIE 2017)

ser diversificada e desenvolvida com a interação em grupo. Contar a história em grupo é aprender pelo contato com outras visões e conhecimentos de algo que alguém participou ou não. A Figura 1 mostra como é constituída uma história, bem como a proposta de associações entre fragmentos dentro da própria história e fragmentos de outras histórias - este último tipo de associação que correlaciona fragmentos de outras histórias não foi abordado neste trabalho.

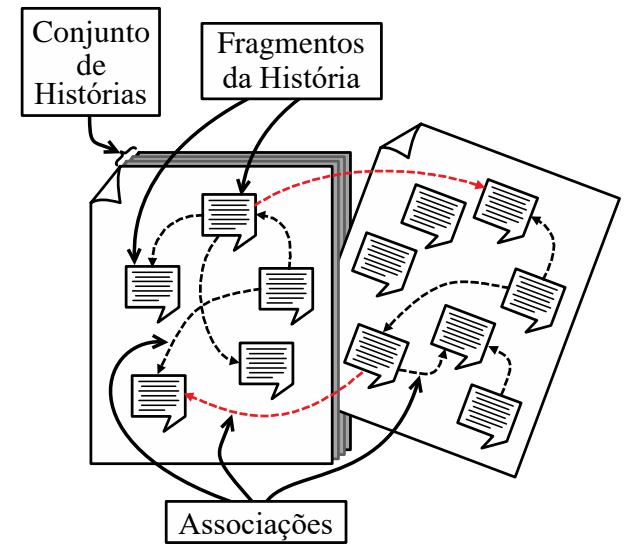

Figura 1. Abstração da estrutura das histórias na técnica de Group Storytelling.

Os fragmentos podem ser descritores (descrevem pessoas ou locais da história); fatos (relatos definitivos que não possuem inverdades, geralmente declarações ou documentos oficiais) ou relatos (narrações de forma pessoal). A Figura 2 expõe as classificações dos fragmentos e das associações aplicadas entre eles. Mais detalhes sobre a técnica podem ser encontrados em Luz (2008).

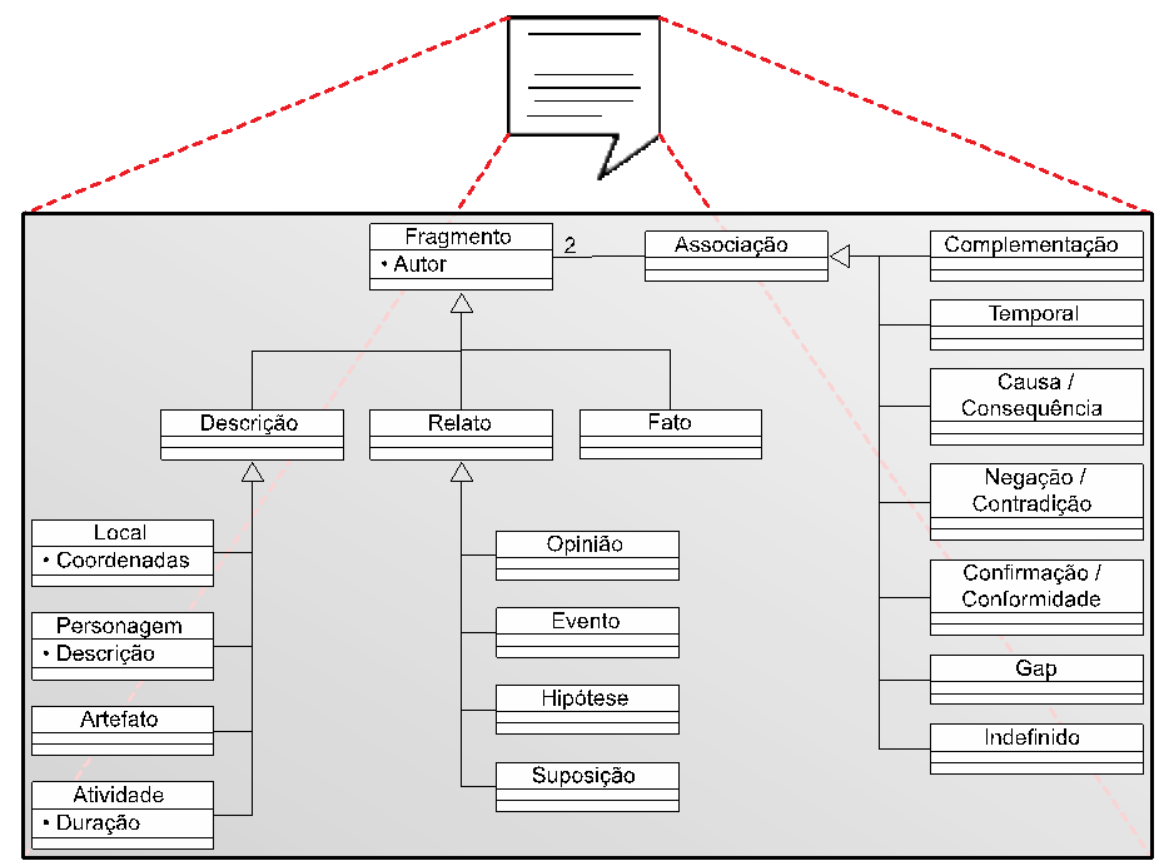

Figura 2. Caracterização de um fragmento e suas associações em uma história no group storytelling. Baseada em Pimentel e Fuks (2012) e Luz (2008).

A ferramenta Sofia 2.0 apoia a técnica de grupo storytelling e seus preceitos e foi escolhida para a condução do experimento que contou com 8 (oito) alunos de graduação 
e 3 (três) facilitadores para a construção da história da disciplina. Este mesmo experimento foi planejado considerando quatro fases (Figura 3). As três primeiras são exatamente as etapas previstas no Quadro 1. Na primeira fase os participantes são convocados e a data/duração do experimento é informada; na segunda são apresentados os conceitos da técnica para os participantes, os objetivos a serem alcançados com o experimento e a construção colaborativa da história através dos fragmentos e seus relacionamentos, bem como os comentários em ambos, na ferramenta computacional de forma individual - ao final desta fase, os facilitadores podem escolher o formato da visualização dos fragmentos que será apresentado aos alunos (ex.: hyperlink, grafo, mapa mental, entre outros) —; já na terceira fase os participantes são divididos aleatoriamente em grupos para a construção de uma versão da história a partir dos fragmentos e relacionamentos criados na Fase II. A quarta fase destina-se à análise das histórias construídas pelos grupos com o objetivo de investigar a percepção e aceitação dos alunos sobre: (i) os conteúdos apresentados ao longo da disciplina, (ii) a forma de condução das aulas e (iii) o método colaborativo de ensino, através da técnica de group storytelling.

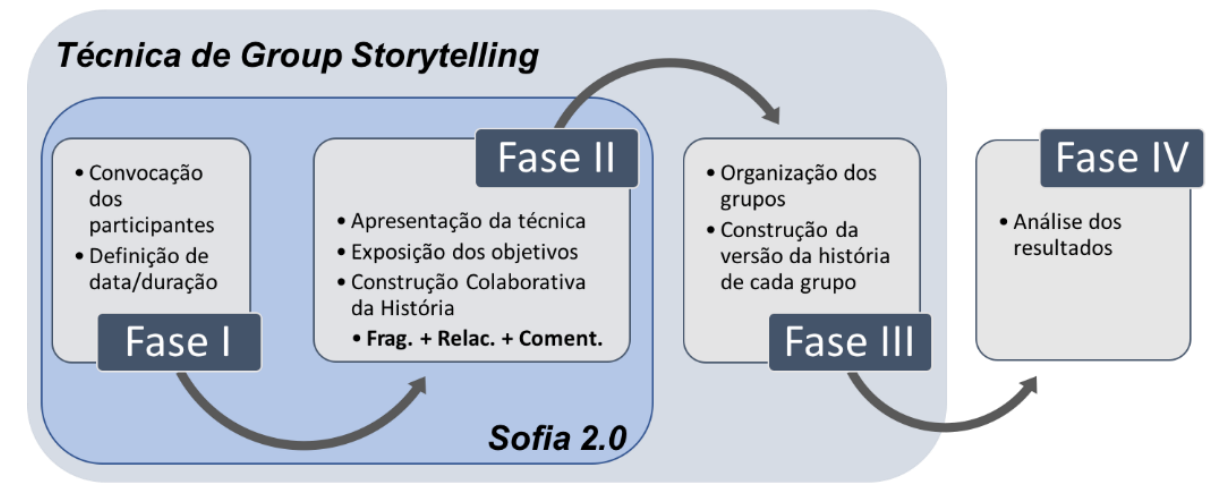

Figura 3. Metodologia de investigação.

Foi solicitado aos contadores da história que eles relatassem os temas abordados na disciplina, fatores motivacionais, o que os desagradou, o que mais chamou a atenção e quais as impressões sobre a condução das aulas e do método de ensino vivenciado por eles. As Fases I e II do experimento ocorreram em sala de aula. A Fase II teve duração de duas horas para ambientação dos participantes à técnica e ferramenta, e mais dois dias para que continuassem a acrescentar fragmentos e relacionamentos na ferramenta Sofia 2.0 (2017), disponibilizada pela universidade e acessível aos participantes. A Fase III teve duração de uma semana onde, ao fim, os grupos apresentaram as histórias na página da disciplina.

Os dados coletados ao longo do experimento foram os fragmentos da história criados e propostos pelos alunos, os relacionamentos entre esses fragmentos, os comentários - todos localizados na ferramenta - e a versão da história sobre a disciplina criada também em grupo pelos alunos. Ao total, foram 4 (quatro) histórias, uma vez que a turma foi dividida em quatro grupos. A Figura 4 apresenta alguns fragmentos e seus relacionamentos coletados no experimento, omitindo, porém, os comentários para não dificultar visualização dos fragmentos e mascarando o nome dos participantes.

Neste experimento optou-se por não utilizar o formato de visualização da história da ferramenta por questões de tempo para a conclusão de outras tarefas da 
VI Congresso Brasileiro de Informática na Educação (CBIE 2017)

Anais do XXIII Workshop de Informática na Escola (WIE 2017)

disciplina, pois os alunos ainda estavam em período de adaptação (Figura 5). Elegeu-se, portanto, como substituto, um mapa conceitual utilizando a ferramenta CMap (2017), que contemplava plenamente, para a visualização, a estrutura da história na técnica de group storytelling. Os comentários eram exibidos ao parar o cursor sobre um fragmento ou relacionamento.

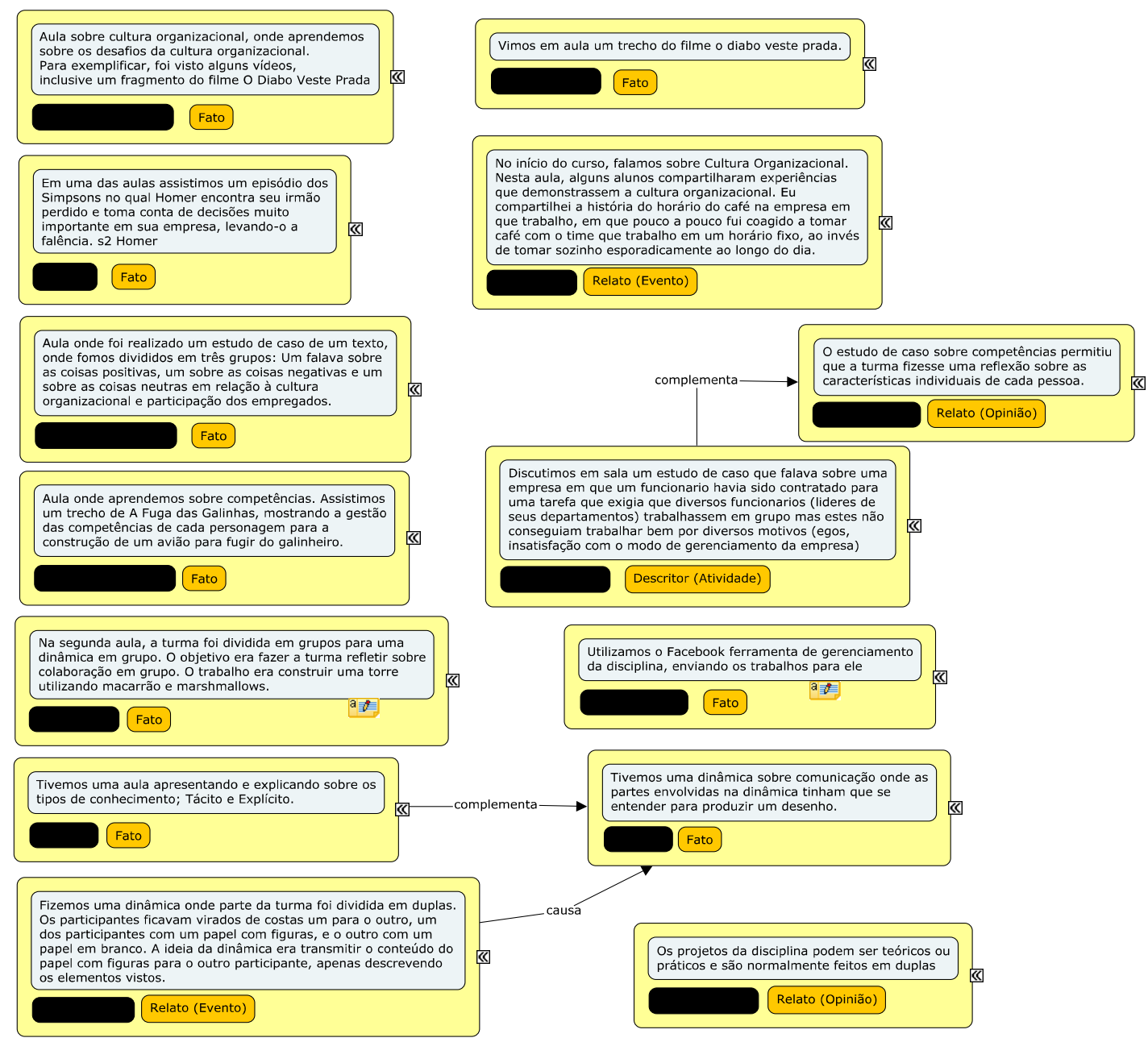

Figura 4. Alguns fragmentos da história da disciplina e seus relacionamentos.

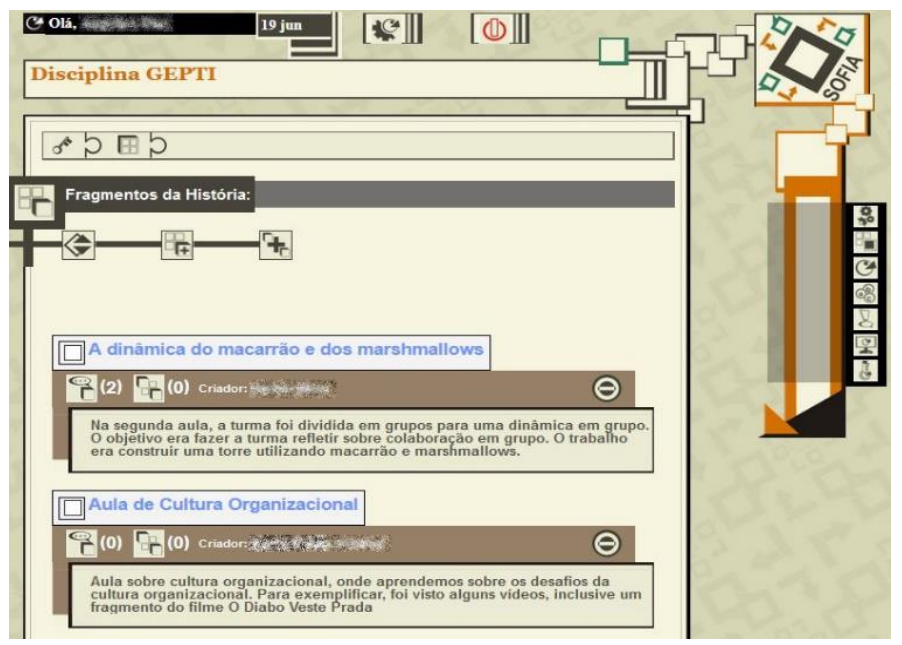

Figura 5. Visualização dos fragmentos na Ferramenta Sofia 2.0. 
Foram ao todo 39 (trinta e nove) fragmentos, dentre os quais 17 (dezessete) fatos que definem argumentos ditos conclusivos, 14 (quatorze) relatos representando opiniões dos alunos, 3 (três) relatos representando descrição de eventos ocorridos, 2 (dois) descritores de atividades que foram conduzidas, $\mathbf{2}$ (dois) descritores delineando algum personagem citado na história e 1 (um) descritor de um local ou acomodação. As associações somaram 15 (quinze), sendo 4 (quatro) do tipo causal, em que um fragmento causa o outro e $\mathbf{1 1}$ (onze) de complementação, onde um fragmento complementa de algum modo o que o outro está descrevendo.

\subsection{Discussão dos resultados}

Os resultados foram analisados por exame do material coletado da ferramenta, das versões escritas das histórias entregues, e pelo crivo de todo esse conteúdo à luz da percepção dos facilitadores - dentre os quais estavam os docentes da disciplina. Esta análise compõe a Fase IV (Figura 3) da metodologia de investigação desta pesquisa e teve duração de duas semanas. Foram levados em conta a dinâmica (participação e contribuições) de cada aluno para a construção da história e a forma como cada grupo escreveu sua versão da história. Dentro deste último, buscou-se o relato dos conceitos dados em sala de aula, mesmo que não pautados nas nomenclaturas técnicas, associação entre os conteúdos, atividades aplicadas em cada conteúdo, exercícios e análises próprias (opiniões, críticas, elogios, sugestões, etc.).

Pode-se destacar dentre os fragmentos coletados que alguns alunos, concentraram-se em determinar fatos referentes aos temas abordados ("Tivemos uma aula apresentando e explicando sobre os tipos de conhecimento; Tácito e Explícito."), outros procuravam complementar com as atividades abordadas ("Tivemos uma dinâmica sobre comunicação onde as partes envolvidas na dinâmica tinham que se entender para produzir um desenho."), outros ainda com detalhes comportamentais dos membros durante as aulas ("Aulas no horário das 8 h costumam ser um problema para alunos, portante [sic] é muito comum vários estarem atrasados (alô [nome aluno]) ou mesmo faltarem."), outros com aspectos contextuais do ambiente ("Usamos na maioria das aulas um projetor que tem defeito."), etc.

A história criada ao fim, refletiu como a disciplina foi conduzida e expôs de modo superficial os conteúdos apresentados ao longo do curso e os objetos de aprendizagem que mais se destacaram para os alunos formuladores dos fragmentos, como os vídeos usados para ilustrar o tema e fomentar discussões, a página da disciplina, as dinâmicas propostas, debates sobre estudos de caso, etc. Porém, poucas conclusões sobre a condução da disciplina, sob a perspectiva do método colaborativo de ensino, foram extraídas das histórias construídas pelos grupos de alunos. Fatos sobre a aprendizagem colaborativa durante o curso foram destacados nas histórias e seus fragmentos, com base nos fragmentos produzidos pela execução do group storrytelling, como: “... parte das aulas são bate-papos onde as professoras frequentemente trazem histórias envolvendo suas experiências e as contam ... para exemplificar os conceitos aprendidos... . Isso também faz com que os alunos gerem discussões sobre diversos temas variados ligando com o conteúdo da disciplina."). Neste trecho fica claro que os alunos entendem o benefício de agregar a experiência do grupo (alunos e professores) na apresentação e discussão das teorias que compõem a grade curricular da disciplina. No entanto, o experimento realizado nesta investigação trouxe poucos exemplos sobre este aspecto. 
VI Congresso Brasileiro de Informática na Educação (CBIE 2017)

Anais do XXIII Workshop de Informática na Escola (WIE 2017)

\section{Conclusões}

Este artigo propôs a utilização de histórias contadas colaborativamente como meio de extração das experiências e percepções dos alunos de uma disciplina como meio de avaliação intermediária dos conteúdos por eles apreendidos através da técnica de group storytelling apoiada pela ferramenta Sofia 2.0. Apesar do pouco contato e a inexperiência com a técnica, a participação dos alunos na dinâmica foi considerada satisfatória, principalmente na análise e classificação dos conceitos passados na disciplina. A maior parte dos alunos teve, inicialmente, dificuldade em externalizar o conhecimento, mas passaram a se sentir confiantes a contribuir com novos fragmentos a medida que leram os fragmentos dos outros alunos e estabeleceram as relações entre eles. Apesar disso, os alunos conseguiram atender aos objetivos da atividade, trabalharam colaborativamente e compreenderam como a técnica apoia na externalização do conhecimento.

Os resultados deste experimento trazem indícios satisfatórios sobre a aplicação da técnica group storytelling no exercício da coleta das percepções e aceitações dos alunos sobre: os conteúdos apresentados ao longo da disciplina, a forma de condução das aulas e o método colaborativo de ensino. Além disso, mostrou-se viável como instrumento de avaliação, mas é aconselhável explica-la desde as primeiras aulas, dando tempo de adaptação aos conceitos da técnica. Apesar de o grupo de alunos ser pequeno, muitos usaram relatos como base de explanação, pouco associaram de forma consistente os conteúdos. A postura assumida por eles foi a de uma tarefa e não a avaliação total deles em relação à disciplina. O ideal é aplica-la desde o início e permitir que os próprios alunos, com o amadurecimento e entendimento dos conceitos refinem a história da disciplina. Com isso, faz parte dos próximos passos desta pesquisa conduzir novos experimentos em turmas distintas a fim de generalizar os resultados alcançados. Ainda como próximos passos, espera-se introduzir esse mecanismo de avaliação como fonte para levantamentos e análises sobre os aspectos positivos ou negativos da disciplina e discuti-los diretamente com alunos e buscar construir colaborativamente possíveis caminhos para a disciplina.

\section{Referências}

Acosta, C. E., Collazos, C. A., Guerrero, L. A., et al. (2004). StoryMapper: A Multimedia Tool to Externalize Knowledge. In Proceedings, XXIV international conference of the Chilean Computer Science Society (SCCC 2004).

CMap. Home - Cmap. http://cmap.ihmc.us/, [Acesso em jun 2017]

Fink, L. D. (2013). Creating significant learning experiences: an integrated approach to designing college courses. Revised and updated edition ed. San Francisco: JosseyBass.

Guterres, J. P. D. e Silveira, M. S. (2016). Analisando o cenário brasileiro de pesquisa de objetos de aprendizagem. In XXVII Simpósio Brasileiro de Informática na Educação (SBIE 2016).

Haigh, C. e Hardy, P. (2011). Tell me a story - a conceptual exploration of storytelling in healthcare education. Nurse Education Today, v. 31, n. 4, p. 408-411. 
VI Congresso Brasileiro de Informática na Educação (CBIE 2017)

Anais do XXIII Workshop de Informática na Escola (WIE 2017)

Krom, C. L. e Williams, S. V. (2011). Tell me a story: Using creative writing in introductory accounting courses to enhance and assess student learning. Journal of Accounting Education, v. 29, n. 4, p. 234-249.

Lage, B. B. e Quintanilha, C. C. (2010). Sofia 2.0: A maturação de um arcabouço para soluções de group storytelling através de uma instanciação avant-garde. Projeto Final - DCC, UFRJ, RJ.

Luz, C. M. (2008) Sofia - Um arcabouço para o desenvolvimento de ferramentas de group storytelling. Dissertação (Mestrado em Informática) - IM/NCE, UFRJ, RJ.

Molina, A. I., Redondo, M. A., Lacave, C. e Ortega, M. (2014). Assessing the effectiveness of new devices for accessing learning materials: An empirical analysis based on eye tracking and learner subjective perception. Computers in Human Behavior, v. 31, p. 475-490.

Padilla-Zea, N., Gutiérrez, F. L., López-Arcos, J. R., Abad-Arranz, A. e Paderewski, P. (2014). Modeling storytelling to be used in educational video games. Computers in Human Behavior, v. 31, p. 461-474.

Pimentel, M. and Fuks, H. (2012). Sistemas colaborativos.

Rambe, P. e Mlambo, S. (2014). Using digital storytelling to externalise personal knowledge of research processes: The case of a Knowledge Audio repository. The Internet and Higher Education, v. 22, p. 11-23.

Salim, R; Dias, a. F.S., França, J.B., 1, Lage, B., Borges, M.R. O uso de storytelling para apoiar o compartilhamento de conhecimento. In Congresso Brasileiro de Informática e Educação (CBIE 2016).

Santos Green, L. (2013). Language learning through a lens: The case for digital storytelling in the second language classroom. School Libraries Worldwide, v. 19, n. 2, p. 23-36.

Sax, B. (2006). Storytelling and the "information overload." On the Horizon, v.14, n.4, p.165-170.

Sofia 2.0. http://labemergencia.ddns.com.br:8090/sofia2.0/, [Acesso em jun 2017]

Souza, A. e Schneider, H. (2016). Aprendizagem colaborativa e conectivismo pedagógico no Facebook. In XXII Workshop de Informática na Escola (WIE 2016).

Tekkumru Kisa, M. and Stein, M. K. (2015). Learning to See Teaching in New Ways: A Foundation for Maintaining Cognitive Demand. American Educational Research Journal, v. 52, n. 1, p. 105-136.

Valle, C., Prinz, W. e Borges, M. (2002). Generation of group storytelling in postdecision implementation process. In The 7th International Conference on Computer Supported Cooperative Work in Design. 